\title{
Estilos de vida asociados al riesgo cardiovascular global en trabajadores universitarios del Estado de México
}

\author{
Patricia Cerecero, M en C,(1) Bernardo Hernández, Dr en C, ${ }^{(2)}$ Dalia Aguirre, Dr en Psic, ${ }^{(1)}$ \\ Roxana Valdés, Dr en C,(I) Gerardo Huitrón, Esp TO.(1)
}

\section{Cerecero P, Hernández B, Aguirre D, Valdés R, Huitrón G. Estilos de vida asociados al riesgo cardiovascular global en trabajadores universitarios del Estado de México. Salud Publica Mex 2009;5 I:465-473.}

\section{Resumen}

Objetivo. Evaluar la asociación del estilo de vida con el riesgo cardiovascular (RCV) en trabajadores universitarios del Estado de México. Material y métodos. Estudio de casos y controles anidado en una cohorte. Se evaluaron variables del estilo de vida, sociodemográficas, antropométricas y antecedentes familiares. El análisis estimó razones de momios pareadas crudas y ajustadas a través de regresión logística condicional. Resultados. Se estudiaron 342 casos con RCV y 684 controles. En los trabajadores con sobrepeso u obesidad, el RCV superó al de aquéllos con peso normal. Los antecedentes familiares de infarto del miocardio se asociaron directamente, y la actividad física moderada-vigorosa inversamente con el RCV, en modelos con interacciones entre género y actividad física; esta relación se mantuvo sólo en los hombres. Conclusiones. Los resultados muestran el papel preponderante de la actividad física moderada-vigorosa como factor del estilo de vida asociado con menor RCV.

Palabras clave: riesgo; enfermedades cardiovasculares; dieta; actividad física; estilo de vida; México
Cerecero P, Hernández B,Aguirre D,Valdés R, Huitrón G. Lifestyle conditions related to global cardiovascular risk among university workers in the State of Mexico.

Salud Publica Mex 2009;5 I:465-473.

\section{Abstract}

Objective. To assess the relationship between lifestyle and cardiovascular risk (CR) among university workers in the State of Mexico. Material and Methods. A nested casecontrol study was conducted and lifestyle, sociodemographic, anthropometric, body mass index and family history of cardiovascular disease were assessed. The analysis included the estimation of crude and adjusted odds ratios (OR) using conditional logistic regression. Results. The study included 342 workers with CR and 684 controls. CR was greater for workers with overweight or obesity than for those with normal weight. Family history of myocardial infarction was directly associated with CR, while physical activity was inversely associated. In models with interactions of gender and physical activity, this relation was observed only for men. Conclusions. Results show an inverse association between the lifestyle factor of physical activity and CR.

Key words: risk; cardiovascular diseases; diet; physical activity; lifestyle; Mexico

(I) Universidad Autónoma del Estado de México. Toluca, Estado de México, México.

(2) Instituto Nacional de Salud Pública. Cuernavaca, Morelos, México.

Fecha de recibido: 6 de marzo de 2009 - Fecha de aceptado: 4 de agosto de 2009

Solicitud de sobretiros: Dra. Dalia María Aguirre Pérez. Jesús Carranza 200, col. Universidad. 50000, Toluca, México. México. Correo electrónico: dmaguirrep@uaemex.mx 
$\mathrm{E}^{\mathrm{n}}$ México, las enfermedades cardiovasculares han mostrado un crecimiento exponencial en las últimas dos décadas, ${ }^{1}$ hasta constituir la primera causa de muerte en la población adulta. ${ }^{2,3}$ La Encuesta Nacional de Salud y Nutrición $2006^{4}$ (ENSANUT 2006) muestra la prevalencia de marcadores de enfermedad cardiovascular (ECV) en adultos: diabetes mellitus, 13.5\%; tabaquismo, $10 \%$ en mujeres y $30 \%$ en hombres; hipertensión arterial, 26.5\%; hipercolesterolemia, $26.5 \%$; sobrepeso y obesidad (índice de masa corporal $\geq 25 \mathrm{~kg} / \mathrm{m}^{2}$ ), $70 \%$.

En la actualidad se acepta de manera amplia que el desarrollo y progreso de las enfermedades crónicas están estrechamente relacionados con factores del estilo de vida. ${ }^{5-7}$ La combinación de una dieta poco saludable, inactividad física y factores como el consumo de tabaco y la ingestión excesiva de alcohol tiene un efecto acumulativo, o incluso sinérgico, que propicia una mayor incidencia de enfermedades crónicas. Estudios poblacionales revelan que hasta $80 \%$ de los casos de cardiopatía coronaria y $90 \%$ de los casos de diabetes mellitus tipo 2 pueden evitarse si se adoptan estilos de vida saludables. ${ }^{7,8}$

El alarmante aumento de la obesidad en las últimas décadas en todo el mundo coloca a la mala nutrición por exceso en un primer plano como determinante de enfermedades crónicas. ${ }^{9}$ La alimentación rica en energía y la inactividad física son algunos de los factores del desequilibrio energético entre consumo y gasto que ocasiona el desarrollo de la obesidad. ${ }^{10}$ El exceso de tejido adiposo, en particular en la región abdominal, se ha relacionado con la agrupación de factores de riesgo que constituyen el síndrome metabólico (hipertensión arterial, dislipidemia y glucosa en ayuno alterada) y cuya presencia confiere mayor riesgo de diabetes mellitus tipo 2 y ECV. ${ }^{11-13}$

Es en este contexto que la inactividad física se reconoce como un factor de riesgo cada vez más relevante en la $E C V,{ }^{14,15}$ que contribuye a la morbilidad y la mortalidad prematura por enfermedades crónicas. En cambio, la actividad física frecuente puede reducir o eliminar el riesgo de sufrir ECV, diabetes mellitus tipo $2,{ }^{16}$ osteoporosis, cáncer de colon y cáncer de mama. ${ }^{17}$ En México se han encontrado bajos niveles de actividad física en diversos grupos. ${ }^{4,18}$

Por otro lado, en lo que se refiere a la estratificación del riesgo, el RCV global define la probabilidad de padecer en un periodo de 10 años un evento cardiovascular (infarto del miocardio, angina de pecho, muerte coronaria). Dicho parámetro se calcula mediante las tablas por categorías de factores de riesgo de Wilson, ${ }^{19}$ elaboradas con los datos que arrojó el estudio Framingham, en el cual el riesgo se determina a partir de: la edad (30-74 años), tabaquismo (sí, no), diabetes (sí, no) y las anoma- lías siguientes: lipoproteínas de alta densidad (HDL) bajas, colesterol total (o lipoproteínas de baja densidad) alto y tensión arterial sistólica y diastólica elevada. De acuerdo con el consenso 2001 de ILIB-Latinoamérica, ${ }^{20}$ el riesgo se clasifica en latente $(<10 \%)$, intermedio $(\geq 10 \%$ $\mathrm{y}<20 \%)$ y alto $(\geq 20 \%)$.

Si se considera que los estudios sobre los estilos de vida determinantes del RCV se han realizado de manera primordial en la población anglosajona o caucásica, se advierte la necesidad de investigar estos factores en la población mexicana, ya que son claras las diferencias culturales, genéticas y ambientales con estos grupos. En el presente trabajo se analizó la relación entre el RCV global y los factores del estilo de vida en trabajadores de la Universidad Autónoma del Estado de México (UAEMex).

\section{Material y métodos}

Se diseñó un estudio de casos y controles anidado en la "Cohorte de trabajadores de la UAEMex, 2004". Este estudio forma parte de una investigación interinstitucional en la que participan el Instituto Mexicano del Seguro Social, el Instituto Nacional de Salud Pública (INSP) y la UAEMex. Este protocolo pretende probar ciertas hipótesis acerca de la relación entre los estilos de vida y la incidencia de algunas enfermedades crónicas de relevancia epidemiológica en México. La metodología empleada se ha publicado ya con anterioridad. ${ }^{21,22}$

La población universitaria consta de 7405 trabajadores (mujeres, 41\%; hombres, 59\%) de todas las categorías laborales: administrativos, docentes, investigadores, personal médico, de conservación e intendencia, residentes de 13 localidades del Estado de México. La muestra se conformó con 2555 trabajadores que participaron en la medición basal, en su mayoría (85\%) habitantes de la ciudad de Toluca.

Para el presente estudio se determinó de forma inicial el RCV a través de la metodología que propuso Wilson, ${ }^{19}$ con base en los siguientes factores: edad, colesterol, HDL, tensión arterial, glucosa $\geq 110 \mathrm{mg} / 100 \mathrm{ml}$ o diagnóstico previo de diabetes mellitus y tabaquismo. Con esta metodología se construye un puntaje de riesgo individual, que se traduce en un porcentaje de acuerdo con valores establecidos en tablas.

Se definió como caso a todo trabajador participante de la cohorte con $R C V \geq 10 \%$ y como control a todo trabajador con RCV $<10 \%$, con lo cual se obtuvo un total de 380 casos (15\%). Del total de la muestra se excluyeron 317 observaciones con consumos de energía biológicamente no plausibles $(<500$ o $>7000 \mathrm{kcal} /$ día), 38 de los cuales fueron casos. No se identificaron diferencias significativas en el valor promedio del porcentaje de 
RCV entre los casos excluidos y los casos considerados en el análisis. Los 342 casos restantes se parearon por género con dos controles seleccionados por muestreo sistemático.

Los datos de la dieta y consumo de alcohol se obtuvieron de un cuestionario semicuantitativo de frecuencia de consumo de alimentos y bebidas, validado en la población mexicana. ${ }^{23}$ Se preguntó acerca de la frecuencia de consumo de una porción estándar de 116 alimentos y ocho tipos diferentes de bebidas durante el último año, de acuerdo con 10 opciones de respuesta (desde nunca, hasta seis o más por día) y 11 opciones de respuesta en la cantidad de consumo de bebidas (desde cero hasta más de 15 copas). El cálculo del consumo de nutrimentos específicos se estimó con el programa de cómputo SNUT* desarrollado por el INSP. Los componentes de la dieta analizados incluyeron el consumo de energía total, proteínas, hidratos de carbono y lípidos.

El nivel de actividad física se evaluó con un cuestionario diseñado para calcular la intensidad (ligera, moderada o intensa) y la duración de diferentes tipos de actividad física en una semana típica en el último año, tras considerar sólo aquellas que generan un gasto energético $\geq 3.5$ equivalentes metabólicos (MET) ${ }^{24}$ (caminar, correr, andar en bicicleta, jugar futbol, etc.), con siete opciones de respuesta (desde cinco minutos hasta más de seis horas por semana) y se clasificaron de acuerdo con las recomendaciones de la Organización Panamericana de la Salud ${ }^{9}$ (OPS) en: actividad recomendada $(\geq 3 \mathrm{~h} / \mathrm{sem})$, actividad insuficiente $(<3 \mathrm{~h} /$ sem) e inactividad (ninguna actividad). Además, como indicador de inactividad física se consideró el tiempo dedicado a actividades de bajo consumo energético (<3.5 MET), como leer, escribir, usar la computadora, ver televisión, etc. Mediante cuestionario se obtuvo información sobre: género, años de escolaridad y antecedentes familiares de hipertensión arterial, infarto del miocardio y diabetes mellitus.

El peso corporal y la talla se midieron con ropa ligera y sin zapatos con balanzas electrónicas y estadímetros convencionales. Se calculó el índice de masa corporal (IMC) al dividir el peso corporal en kilogramos entre el cuadrado de la estatura en metros. Con este índice, los trabajadores se agruparon de acuerdo con la clasificación de la Organización Mundial de la Salud ${ }^{25}$ (OMS) en: peso normal (IMC $\leq 24.9$ ), sobrepeso (IMC, 25 a 29.9) y obesidad (IMC $\geq 30$ ). En la medición de la tensión arterial se empleó un monitor automático digi-

\footnotetext{
* Instituto Nacional de Salud Pública, México. SNUT 2.1: sistema para cálculo de vectores nutricionales. México, 1996.
}

tal modelo $\mathrm{CH}$ 656C. Los participantes permanecieron sentados, con el brazo derecho apoyado sobre una mesa y el pliegue anterior del codo a nivel del corazón. Se efectuaron tres determinaciones con intervalos de dos minutos y se registró el promedio.

Para la aplicación de las encuestas, se convocó a los trabajadores a una reunión en su centro de adscripción en los diferentes planteles de la UAEMex, donde se aplicó un cuestionario autoadministrado, previa explicación de los objetivos del estudio y firma del consentimiento informado. Una vez recuperadas las encuestas, se citó a los participantes en las instalaciones del Centro de Investigación en Ciencias Médicas (CICMED-UAEMex) con la indicación de presentarse con ropa ligera, en ayuno de por lo menos 12 horas, sin haber realizado actividad física intensa o haber ingerido alcohol. Personal capacitado y estandarizado llevó a cabo las medidas antropométricas, la toma de tensión arterial y muestras sanguíneas, que de inmediato se centrifugaron y procesaron con un equipo automatizado para determinar concentraciones plasmáticas de glucosa, HDL y colesterol. Los cuestionarios se capturaron con un lector óptico. El Comité de Investigación y Ética del CICMED-UAEMex aprobó los procedimientos empleados.

Los datos se analizaron con el paquete estadístico STATA VII. El análisis descriptivo consistió en el cálculo de media y desviación estándar en las variables continuas (dieta) y de frecuencias simples y proporciones en las variables categóricas. Se buscó la asociación entre el $\mathrm{RCV}$, dieta y actividad física tras comparar los casos y los controles a través de pruebas de $\chi^{2}$ en las variables categóricas y regresión lineal ajustando por el efecto de pareamiento y por el efecto de agrupamiento (por plantel de la universidad) en las variables continuas. Se ajustó por energía total el consumo de los nutrimentos mediante el método de residuales. En este método se ajustan modelos de regresión lineal con el consumo energético como la variable independiente y el consumo de los nutrimentos absolutos como variables dependientes, y se estimaron los residuales para tener una medida del consumo de cada nutrimento al remover el efecto del consumo de energía. ${ }^{26}$ Se estimaron razones de momios pareadas crudas e intervalos de confianza exactos al 95\% ajustados por género. Con modelos de regresión logística condicionada e interacciones se calcularon las razones de momios ajustadas.

\section{Resultados}

La muestra de estudio incluyó a 1026 trabajadores, $30.7 \%$ mujeres y $69.3 \%$ hombres, con una edad media de 45.4 años. La probabilidad promedio de desarrollar un episodio cardiovascular fue $8.3 \%$ (hombres, $8.8 \%$; 
mujeres, 7.2\%). En promedio, la edad de los casos (51.7 años) fue superior a la de los controles (42.3 años).

Las características de la muestra se presentan en el cuadro I. Respecto del IMC, 27.5\% mostró peso normal y $72.5 \%$ sobrepeso u obesidad. Poco más de una tercera parte $(36.4 \%)$ manifestó no realizar actividad física y más de cuatro quintas partes consumir al menos una bebida alcohólica por día (87.5\%). Cerca de la mitad de los participantes refirió antecedentes familiares (AF) de hipertensión y diabetes mellitus, además de haber cursado entre 14 y 17 años de estudio (46.2\%).

Se observó una mayor proporción de trabajadores con sobrepeso $(57.0 \%) \mathrm{u}$ obesidad $(24.6 \%)$ en los casos respecto de los controles $(\rho<0.001)$. Asimismo, los AF de infarto del miocardio e hipertensión en los casos superaron a los de los controles. No se observaron diferencias significativas en los años de escolaridad, las horas por semana dedicadas a actividades moderadasvigorosas o de bajo consumo energético y el consumo por día de energía total, nutrimentos y bebidas alcohólicas (cuadro II).

Se calcularon razones de momios pareadas $(R M p)$, crudas y ajustadas, de RCV por IMC y estilos de vida. En el análisis crudo se observó mayor RCV en los individuos con obesidad ( $R M p=2.62$, IC95\%: $1.75-3.91 \mathrm{~kg} /$ $\mathrm{m}^{2}$ ) o sobrepeso $\left(R M p=1.85, I C 95 \%: 1.33-2.58 \mathrm{~kg} / \mathrm{m}^{2}\right)$ que en aquéllos con peso normal. En los individuos que realizan actividades moderadas-vigorosas durante tres o más horas por semana, el RCV fue $29 \%$ menor comparado con el de quienes dedican menos de tres horas por semana o son físicamente inactivos ( $R M p=0.71, I C 95 \%$ : $0.51-0.98 \mathrm{~h} / \mathrm{sem}$ ). El análisis con RMp ajustadas por IMC y AF de infarto del miocardio e hipertensión reveló que el sobrepeso, la obesidad y tres o más horas por semana de actividad física conservaron su significancia estadística. No se observaron asociaciones significativas entre las actividades de bajo consumo energético, el consumo de bebidas alcohólicas o macronutrimentos con el RCV (cuadro III).

Los resultados del análisis por género se muestran en el cuadro IV. La prevalencia de sobrepeso en el masculino (56.3\%) superó a la del femenino (48.0\%); en cambio, la prevalencia de obesidad en el femenino (23.0\%) fue mayor 6 puntos porcentuales respecto de la del masculino (17.0\%). La proporción de mujeres físicamente inactivas $(52.7 \%$ ) fue 23 puntos porcentuales superior a la de los hombres (29.2\%).

Con RMp de RCV por IMC y estilos de vida, ajustadas por AF de infarto del miocardio e hipertensión, los hombres con sobrepeso tuvieron momios 50\% mayores y los hombres con obesidad momios $100 \%$ mayores de

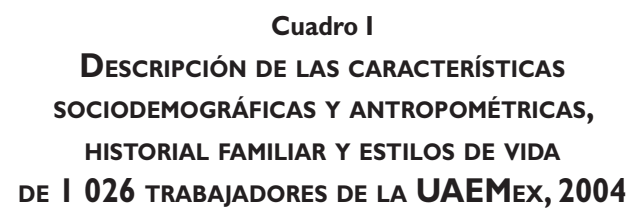

Variable

$\%$

Escolaridad (años)

\begin{tabular}{lll}
$\leq 13$ & 317 & 31.0 \\
\hline $14-17$ & 475 & 46.2 \\
\hline$\geq 18$ & 234 & 22.8
\end{tabular}

IMC $\left(\mathrm{kg} / \mathrm{m}^{2}\right)$

\begin{tabular}{lcc}
$<25$ (peso normal) & 282 & 27.5 \\
\hline $25-30$ (sobrepeso) & 551 & 53.7 \\
\hline 230 (obesidad) & 193 & 18.8
\end{tabular}

Antecedentes familiares

$\begin{array}{lll}\text { Infarto del miocardio } & 214 & 20.9\end{array}$

$\begin{array}{lll}\text { Diabetes mellitus } & 512 & 49.9\end{array}$

Hipertensión

Actividad moderada-vigorosa ( $\geq 3$ MET)

Inactividad $(0 \mathrm{~h} / \mathrm{sem})$

Insuficiente $(<3 \mathrm{~h} / \mathrm{sem})$

$373 \quad 36.4$

Recomendada ( $\geq 3 \mathrm{~h} / \mathrm{sem})$

$\begin{array}{ll}316 & 30.8 \\ 337 & 32.8\end{array}$

Actividad de bajo consumo energético ( $\mathrm{h} / \mathrm{sem})$

\begin{tabular}{lll}
$<11$ & 334 & 32.5 \\
\hline $1 \mid-22$ & 361 & 35.2 \\
\hline$>22$ & 331 & 32.3
\end{tabular}

Bebidas alcohólicas (copas/día)

\begin{tabular}{|c|c|c|}
\hline 0 & 128 & 12.5 \\
\hline$<2$ & 861 & 83.9 \\
\hline$\geq 2$ & 37 & 3.6 \\
\hline Consumo diario & Media & $\mathrm{DE}^{*}$ \\
\hline Energía total (kcal) & 2283 & 972 \\
\hline Lípidos ${ }^{\ddagger}$ & 61.0 & 15.8 \\
\hline Proteínas ${ }^{\ddagger}$ & 80.5 & 17.5 \\
\hline Hidratos de carbono ${ }^{\ddagger}$ & 343.1 & 114.0 \\
\hline
\end{tabular}

Lípidos, proteínas e hidratos de carbono ajustados por energía total, 


\section{Cuadro II}

COMPARACIÓN DE LAS CARACTERÍSTICAS SOCIODEMOGRÁFICAS Y ANTROPOMÉTRICAS, HISTORIAL FAMILIAR Y ESTILOS DE VIDA DE TRABAJADORES DE LA UAEMEX, CON Y SIN RIESGO CARDIOVASCULAR, 2004

$\begin{array}{ccc}\% \text { Casos } & \% \text { Controles } \\ \text { Variable } & n=342 \quad n=684 \quad p^{*}\end{array}$

Escolaridad (años)

\begin{tabular}{llll}
$\leq 13$ & 30.7 & 31.0 & \\
\hline $14-17$ & 45.0 & 47.0 & \\
\hline$\geq 18$ & 24.3 & 22.0 & 0.717
\end{tabular}

IMC $\left(\mathrm{kg} / \mathrm{m}^{2}\right)$

\begin{tabular}{llll}
$<25$ (peso normal) & 18.4 & 32.0 & \\
\hline $25-30$ (sobrepeso) & 57.0 & 52.0 & \\
\hline$\geq 30$ (obesidad) & 24.6 & 16.0 & $<0.001$
\end{tabular}

Antecedentes familiares

\begin{tabular}{cccc} 
Infarto del miocardio & 24.6 & 19.0 & 0.039 \\
\hline Diabetes mellitus & 50.0 & 49.8 & 0.965 \\
\hline Hipertensión & 55.5 & 48.9 & 0.047
\end{tabular}

Actividad moderada-vigorosa

\begin{tabular}{llll} 
Inactividad $(0 \mathrm{~h} / \mathrm{sem})$ & 39.4 & 34.8 & \\
\hline Insuficiente $(<3 \mathrm{~h} / \mathrm{sem})$ & 31.9 & 30.2 & \\
\hline Recomendada $(\geq 3 \mathrm{~h} / \mathrm{sem})$ & 28.7 & 35.0 & 0.116
\end{tabular}

Actividad bajo consumo energetico

\begin{tabular}{llll}
$<1 \mathrm{l} \mathrm{h} / \mathrm{sem}$ & 34.2 & 31.8 & \\
\hline $1 \mathrm{I}-22 \mathrm{~h} / \mathrm{sem}$ & 35.1 & 35.2 & \\
\hline$>22 \mathrm{~h} / \mathrm{sem}$ & 30.7 & 33.0 & 0.663
\end{tabular}

Bebidas alcohólicas

\begin{tabular}{|c|c|c|c|}
\hline 0 copas/día & 12.3 & 12.6 & \\
\hline$<2$ copas/día & 83.0 & 84.4 & \\
\hline$\geq 2$ copas/día & 4.7 & 3.1 & 0.428 \\
\hline Consumo diario & Media $(D E)^{\ddagger}$ & Media $(D E)^{\ddagger}$ & \\
\hline Energía total (kcal) & $2219(917)$ & $2316(998)$ & 0.121 \\
\hline Lípidos $\$$ & $60.0(14.9)$ & $61.5(16.3)$ & 0.090 \\
\hline Proteínas ${ }^{\S}$ & $81.3(16.5)$ & $80.2(18.0)$ & 0.507 \\
\hline Hidratos de carbono & $344.2(54.8)$ & $342.6(51.3)$ & 0.593 \\
\hline \multicolumn{4}{|c|}{$\begin{array}{l}\text { * Prueba } \chi^{2} \text { para las variables categóricas, análisis de regresión lineal ajus } \\
\text { tado por el efecto de pareamiento y agrupamiento para las variables } \\
\text { continuas } \\
\text { Desviación estándar } \\
\text { Lípidos, proteínas e hidratos de carbono ajustados por energía total } \\
\text { método de residuales }{ }^{26}\end{array}$} \\
\hline
\end{tabular}

Lípidos, proteínas e hidratos de carbono ajustados por energía total,
Cuadro III

RAZONES DE MOMIOS PAREADAS, CRUDAS

Y AJUSTADAS, DE RIESGO CARDIOVASCULAR DE ACUERDO CON EL IMC Y ESTILOS DE VIDA DE TRABAJADORES DE LA UAEMEX, 2004

Variable RMp cruda IC95\% RMp ajustada* IC95\%

IMC $\left(\mathrm{kg} / \mathrm{m}^{2}\right)$

\begin{tabular}{lcccc}
$\geq 30$ (obesidad) & 2.62 & $\mathrm{I} .753 .9 \mathrm{I}$ & 2.57 & $\mathrm{I} .723 .9 \mathrm{I}$ \\
\hline $25-30$ (sobrepeso) & $\mathrm{I} .85$ & $\mathrm{I} .332 .58$ & $\mathrm{I} .85$ & $\mathrm{I} .693 .89$ \\
\hline$<25$ (peso normal) & $\mathrm{I}$ & & $\mathrm{I}$ &
\end{tabular}

Actividad moderada-vigorosa

\begin{tabular}{|c|c|c|c|c|}
\hline Recomendada ( $\geq 3 \mathrm{~h} / \mathrm{sem})$ & 0.71 & 0.510 .98 & 0.71 & 0.510 .99 \\
\hline Insuficiente (<3 h/sem) & 0.93 & $0.67 \quad I .28$ & 0.96 & $0.68 \mathrm{I} .35$ \\
\hline Inactividad $(0 \mathrm{~h} / \mathrm{sem})$ & I & & I & \\
\hline
\end{tabular}

Act. de bajo consumo energético

\begin{tabular}{lcccc}
$\geq 22 \mathrm{~h} / \mathrm{sem}$ & 0.84 & $0.60 \mathrm{I} .18$ & 0.92 & $0.65 \mathrm{I} .3 \mathrm{I}$ \\
\hline II a $22 \mathrm{~h} / \mathrm{sem}$ & 0.91 & $0.66 \mathrm{I} .25$ & 0.93 & $0.67 \mathrm{I} .30$ \\
\hline$<\mathrm{II} \mathrm{h} / \mathrm{sem}$ & $\mathrm{I}$ & & $\mathrm{I}$ &
\end{tabular}

Bebidas alcohólicas (copas/día)

\begin{tabular}{|c|c|c|c|c|}
\hline$\geq 2$ & 1.51 & 0.703 .28 & 1.34 & 0.563 .23 \\
\hline$<2$ & 0.99 & $0.66 \mid .48$ & 0.90 & $0.59 \quad \mid .38$ \\
\hline
\end{tabular}

Consumo diario

\begin{tabular}{|c|c|c|c|c|}
\hline Lípidos $^{\ddagger}$ & 0.99 & 0.991 .00 & 0.99 & 0.971 .00 \\
\hline Proteínas ${ }^{\ddagger}$ & 1.00 & $0.99 \quad 1.00$ & 1.00 & 0.991 .01 \\
\hline Hidratos de carbono ${ }^{\ddagger}$ & 1.00 & 0.991 .00 & 1.00 & 0.991 .01 \\
\hline
\end{tabular}

* Ajustada por IMC $\left(<25,25-30, \geq 30 \mathrm{~kg} / \mathrm{m}^{2}\right)$ y antecedentes familiares de infarto del miocardio e hipertensión

‡ Lípidos, proteínas e hidratos de carbono ajustados por energía total, método de residuales ${ }^{26}$

RMp: razón de momios pareada, obtenida a través de regresión logística condicional

presentar RCV alto (es decir, ser considerados como casos), en comparación con los hombres con peso normal $(R M p=1.56$, IC95\%: 1.03-2.36; $R M p=2.05$, IC95\%: 1.19 3.52). En las mujeres, la presencia de obesidad triplicó las posibilidades de pertenecer al grupo de los casos en comparación con las mujeres sin obesidad $(R M p=3.30$, IC95\%:1.10-9.88). Además, en los hombres que realizan actividades moderadas-vigorosas durante tres o más horas por semana, las posibilidades de presentar RCV alto fueron $36 \%$ menores que las de los hombres que 


\section{Cuadro IV}

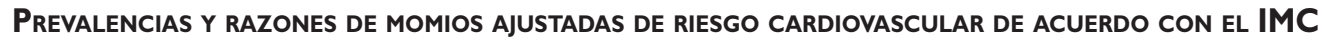
Y ACTIVIDAD FíSICA MODERADA-VIGOROSA, ESTRATIFICADAS POR GÉNERO EN TRABAJADORES DE LA UAEMEX, 2004

\begin{tabular}{|c|c|c|c|c|}
\hline & & & asculino $(n=7$ & \\
\hline & \% Total & $\%$ Casos & \% Controles & RMp ajustada* (IC95\%) \\
\hline IMC (kg/m2) & & & & \\
\hline$\geq 30$ (obesidad) & 17.0 & 21.5 & 14.8 & $2.05(1.19,3.52)$ \\
\hline $25-30$ (sobrepeso) & 56.3 & 59.5 & 54.6 & $1.56(1.03,2.36)$ \\
\hline$<25$ (peso normal) & 26.7 & 19.0 & 30.6 & I \\
\hline Actividad moderada-vigorosa & & & & \\
\hline Recomendada $(\geq 3 \mathrm{~h} / \mathrm{sem})$ & 37.5 & 30.8 & 41.0 & $0.64(0.42,0.96)$ \\
\hline Insuficiente $(<3 \mathrm{~h} / \mathrm{sem})$ & 33.3 & 34.2 & 32.9 & $1.02(0.66,1.55)$ \\
\hline Inactividad $(0 \mathrm{~h} / \mathrm{sem})$ & 29.2 & 35.0 & 26.1 & $\mathrm{I}$ \\
\hline & & & menino $(n=3$ & \\
\hline & \% Total & $\%$ Casos & $\%$ Controles & RMp ajustada* (IC95\%) \\
\hline IMC (kg/m2) & & & & \\
\hline$\geq 30$ (obesidad) & 23.0 & 31.4 & 18.5 & $3.30(1.10,9.88)$ \\
\hline $25-30$ (sobrepeso) & 48.0 & 51.4 & 46.2 & $2.02(0.82,4.97)$ \\
\hline$<25$ (peso normal) & 29.0 & 17.2 & 35.2 & I \\
\hline Actividad moderada-vigorosa & & & & \\
\hline Recomendada $(\geq 3 \mathrm{~h} / \mathrm{sem})$ & 22.2 & 23.8 & 21.4 & $\mathrm{I} .2 \mathrm{I}(0.5 \mathrm{I}, 2.86)$ \\
\hline Insuficiente $(<3 \mathrm{~h} / \mathrm{sem})$ & 25.1 & 26.7 & 24.3 & $\mathrm{I} .4 \mathrm{I}(0.43,4.58)$ \\
\hline Inactividad $(0 \mathrm{~h} / \mathrm{sem})$ & 52.7 & 49.5 & 54.3 & I \\
\hline
\end{tabular}

* Ajustada por antecedentes familiares de infarto del miocardio e hipertensión

RMp: razón de momios pareada, obtenida a través de regresión logística condicional IC $95 \%$ : intervalo de confianza al $95 \%$

dedican menos de tres horas por semana o son físicamente inactivos ( $R M p=0.64$, IC95\%: 0.42-0.96).

Los resultados de modelos de regresión logística condicional para la estimación de RCV por AF de infarto del miocardio e hipertensión, y por la interacción entre género y las horas por semana dedicadas a actividades físicas, se muestran en el cuadro V. Los individuos con AF de infarto del miocardio tuvieron momios $40 \%$ mayores de presentar RCV alto que los que no tuvieron esos $\mathrm{AF}$, al ajustar por género, actividad física moderada-vigorosa por semana, IMC, consumo de bebidas alcohólicas por día y horas por semana dedicadas a actividades de bajo consumo energético ( $R M p=1.41$, IC95\%: 1.00-1.98). Se encontró un efecto protector de la actividad moderada-vigorosa durante tres o más horas por semana en el género masculino. En los hombres con actividad física regular, las posibilidades de presentar $\mathrm{RCV}$ alto fueron 1.8 veces menores (inverso de la razón de momios estimada) tras ajustar por IMC, AF de infar- to del miocardio e hipertensión, consumo de bebidas alcohólicas por día y horas por semana dedicadas a actividades de bajo consumo energético $(R M p=0.56$, IC95\%: 0.37-0.83).

\section{Discusión}

En este estudio en trabajadores de una universidad pública, la prevalencia de RCV global fue $15 \%$, cifra que no pudo compararse con otros datos nacionales debido a que no hay estudios previos disponibles en México sobre el tema. El riesgo promedio de presentar un episodio cardiovascular a 10 años fue $8.3 \%$, menor al encontrado en adultos españoles de 35 a 74 años $(17.0 \%){ }^{27}$ y superior al reportado en adultos colombianos $(3.87 \%) .^{28}$

En los trabajadores con riesgo de ECV, la edad promedio fue superior a la de aquéllos con un riesgo latente. Se sabe que en el adulto existe un incremento del riesgo metabólico y cardiovascular a causa del proceso natural 


\section{Cuadro V}

Modelo de REgRESIÓN LOGíSTICA CONDICIONAL, RIESGO ASOCIADO A LA PREVALENCIA DE RIESGO CARDIOVASCULAR POR GÉNERO Y ACTIVIDAD FÍSICA MODERADA-VIGOROSA EN TRABAJADORES DE LA UAEMEX, 2004

\begin{tabular}{|c|c|c|c|}
\hline Factores & $\begin{array}{c}\text { Modelo I } \\
\text { Género y actividad fisica } \\
\text { RMp (IC95\%)* }\end{array}$ & $\begin{array}{c}\text { Modelo II } \\
\text { I más IMC,AF de infarto } \\
\text { e hipertensión } \\
\text { RMp (IC95\%) }\end{array}$ & $\begin{array}{c}\text { Modelo III } \\
\text { II más consumo de alcohol } \\
\text { y actividad <3.5 MET } \\
\text { RMp (IC95\%)* }\end{array}$ \\
\hline
\end{tabular}

\begin{tabular}{|c|c|c|c|}
\hline $\begin{array}{l}\text { Género } \delta^{\S} \text { actividad física moderada-vigorosa } \\
\text { Femenino }{ }^{\S} \text { actividad recomendada }(n=70)\end{array}$ & $0.80(0,44, \mid, 45)$ & $0.89(0.48,1.64)$ & $0.90(0.49,1.66)$ \\
\hline Femenino ${ }^{\S}$ actividad insuficiente $(n=79)$ & $0.84(0.48,1.48)$ & $0.89(0.50,1.60)$ & $0.91(0.50,1.63)$ \\
\hline Femenino inactividad $(n=166)$ & $0.67(0.43,1.06)$ & $0.7 \mathrm{I}(0.44, \mathrm{I} .14)$ & $0.66(0.4 \mathrm{I}, \mathrm{I} .06)$ \\
\hline Masculino ${ }^{\S}$ actividad recomendada $(n=267)$ & $0.55(0.37,0.82)$ & $0.55(0.37,0.8 I)$ & $0.56(0.37,0.83)$ \\
\hline Masculino $\$$ actividad insuficiente $(n=237)$ & $0.77(0.52,1.14)$ & $0.81(0.54,1.22)$ & $0.82(0.55,1.23)$ \\
\hline Masculino ${ }^{\S}$ inactividad $(n=207)$ & 1 & I & I \\
\hline \multicolumn{4}{|l|}{ AF de infarto del miocardio } \\
\hline Sí $(n=2 \mid 4)$ & & & $1.41(1.00,1.98)$ \\
\hline No $(n=8 \mid 2)$ & & & 1 \\
\hline \multicolumn{4}{|l|}{ AF de hipertensión } \\
\hline Sí $(n=525)$ & & & $1.32(0.98,1.77)$ \\
\hline No $(n=50 \mathrm{I})$ & & & 1 \\
\hline \multicolumn{4}{|c|}{ * Razones de momios pareadas (intervalo de confianza al 95\%) } \\
\hline AF: antecedentes familiares & & & \\
\hline
\end{tabular}

de envejecimiento, la disminución de las capacidades funcionales y la redistribución de la grasa corporal en el área abdominal:; ${ }^{29}$ aunado a esto, los estilos de vida no saludables, como el sedentarismo encontrado en este estudio, contribuyen a la aceleración del proceso ateroesclerótico.

Además de los factores del estilo de vida, un aspecto importante a considerar en la etiología de la ECV es la vulnerabilidad genética. El historial familiar de infarto del miocardio es un factor de riesgo independiente de $\mathrm{ECV} .^{30}$ En este estudio, los antecedentes familiares de infarto del miocardio se relacionaron de forma significativa con el RCV. Un resultado similar se obtuvo en un estudio multicéntrico en cuatro países de América Latina, en el cual la RM de infarto del miocardio en México, de acuerdo con el historial familiar de enfermedad coronaria, fue de $1.46 .{ }^{31}$

La prevalencia combinada de sobrepeso y obesidad en los trabajadores universitarios $(72.5 \%)$ es superior a la reportada por la ENSANUT $2006^{4}$ para población de 30 a 60 años (70.0\%); no obstante, se identificó la misma tendencia nacional de mayor obesidad en mujeres que en hombres.

En los trabajadores con obesidad, la probabilidad de padecer una ECV duplicó a la de aquéllos con peso normal; asimismo, el sobrepeso intensificó el RCV.
Mientras que la obesidad en las mujeres triplicó el riesgo de una ECV, en los hombres la duplicó. En la actualidad se sabe que el tejido adiposo es el centro de una intrincada red reguladora que modula muchas funciones del cuerpo, incluidas la respuesta inmunitaria e inflamatoria, la angiogénesis, la fibrinólisis y la coagulación, entre otras. ${ }^{32}$ Los resultados de este estudio confirman que la obesidad forma parte importante de los factores de riesgo asociados con mayor riesgo de desarrollar ECV.

Por otro lado, en varios países se ha documentado una disminución de la actividad física de la población, debido en parte a la urbanización y a los cambios en los estilos de vida. La OPS ${ }^{9}$ refiere que entre 30 y $60 \%$ de la población no alcanza los niveles recomendados de actividad física. En este estudio, $67.2 \%$ no alcanzó estos niveles recomendados para la reducción de ECV.

La prevalencia de inactividad física en este estudio $(36.4 \%)$ es casi el doble de la obtenida en la encuesta nacional en derechohabientes del IMSS $(16.8 \%)^{33}$ y de la prevalencia mundial promedio en adultos estimada por la OMS (17\%). ${ }^{34}$ Esta alta prevalencia podría explicarse por la relativa homogeneidad en el nivel socioeconómico de la muestra, dado que la mayoría de los universitarios tiene un nivel de escolaridad alto $(69 \%)$ y no se incluyeron participantes de áreas rurales o de extrema 
marginación, sobre todo si se toma en cuenta que la vida sedentaria se relaciona con la urbanización y el desarrollo tecnológico. ${ }^{9}$

Entre las aportaciones del estudio destaca la identificación de la actividad física regular como factor protector independiente de RCV, sobre todo en los hombres, quienes mostraron realizar actividad física con más regularidad que las mujeres. Un resultado similar se obtuvo en un estudio en hombres de 35 a 65 años de edad de Oaxaca, México, en el cual los niveles altos de actividad física fueron un factor protector del sínd rome metabólico. ${ }^{35}$

Una de las limitaciones del estudio radica en la posibilidad de sesgos de memoria, debido a que la información sobre la exposición a factores del estilo de vida se recolectó en forma retrospectiva, lo que puede ocasionar subestimación o sobreestimación de la exposición, especialmente en parámetros de alta variabilidad como es la dieta. Asimismo, cabe la posibilidad de que un gran número de trabajadores accediera a participar en el estudio por tener conocimiento previo de sus factores de riesgo, lo que tuvo como resultado una mayor prevalencia de RCV. No obstante, por tratarse de un estudio de casos y controles, esto no afecta la estimación de asociaciones con otras variables de interés. Por último, dado que se trata de un estudio transversal, no se puede inferir causalidad entre la presencia de RCV y los niveles de actividad.

Los resultados del estudio muestran el papel preponderante que tiene la actividad física como un factor del estilo de vida asociado con menor RCV. De igual modo, sugieren mayor riesgo de presentar enfermedades cardiovasculares en los trabajadores universitarios con sobrepeso, obesidad o AF de infarto del miocardio en comparación con aquéllos con peso normal o sin AF. Esta información resulta de utilidad en la definición de políticas y medidas de prevención de enfermedades cardiovasculares en la población de adultos universitarios con alto riesgo.

\section{Agradecimientos}

Este trabajo es producto del "Proyecto de Tesis Doctoral" de la UAEMex y del Programa Nacional de Posgrado CONACYT y recibió financiamiento de la UAEMex.

\section{Referencias}

I. Rosas M, Lara A, Pastelín G,Velázquez O, Martínez J, Méndez A, et al. Re-encuesta nacional de hipertensión arterial (RENATA): Consolidación mexicana de los factores de riesgo cardiovascular. Cohorte nacional de seguimiento.Arch Cardiol Mex 2005;75(I):96-III.
2.Velázquez O, Barinagarrementería F, Rubio A, Verdejo J, Méndez M, Violante $R$, et al. Morbilidad y mortalidad de la enfermedad isquémica del corazón y cerebrovascular en México 2005. Arch Cardiol Mex 2007;77:31-39.

3. Rosas M, Pastelín G, Martínez J, Herrera-Acosta J,Attie F. Hipertensión arterial en México. Guías y recomendaciones para su detección, control y tratamiento. Arch Cardiol Mex 2004;74:I34-I57.

4. Oláiz-Fernández G, Rivera-Dommarco J, Shamah-Levy T, Rojas R, Villalpando-Hernández S, Hernández-Ávila M, et al. Encuesta Nacional de Salud y Nutrición 2006. Cuernavaca, México: Instituto Nacional de Salud Pública, 2006.

5.Wannamethee S, Shaper A, Whincup P. Modifiable lifestyle factors and the metabolic syndrome in older men: effects of lifestyle changes. J Am Geriatr Soc 2006;54:1909-1914.

6. Schneiderman N. Psychosocial, behavioral, and biological aspects of chronic diseases. J Am Psychol Soc 2004; 13(6):247-25I.

7. Hu F, Manson J, Stampfer M, Colditz G, Liu S, Solomon C, et al. Diet, lifestyle, and the risk of type II diabetes mellitus in women. N Engl J Med 200I;345:790-797.

8. Key TJ,Allen NE, Spencer EA, Travis RC. The effect of diet on risk of cancer. Lancet 2002;360:86I-868.

9. Organización Panamericana de la salud. Estrategia mundial sobre alimentación saludable, actividad física y salud (DPAS). Plan de implementación en América Latina y el Caribe 2006-2007. Washington, DC: OPS, 2006.

10. Bray GA. Etiology and pathogenesis of obesity. Clin Cornerstone 1999;2:I-15

II. Lee M,Aronne LJ.Weight management for type 2 diabetes mellitus: global cardiovascular risk reduction. Am J Cardiol 2007;19:68-79.

12. Martínez-Palomino G,Vallejo M, Huesca C, Álvarez LE, Paredes G, Lerma GC. Factores de riesgo cardiovascular en una muestra de mujeres jóvenes mexicanas. Arch Cardiol Mex 2006;76(4):40I-407.

13. Koh-Banerjee P,Wang Y, Hu F, Spiegelman D, Willett W, Rimm E. Changes in body weight and body fat distribution as risk factors for clinical diabetes in US men.Am J Epidemiol 2004;159:1 I50-I I59.

14. Kamphius M, Geerlings M, Tijhuis M, Giampaoli S, Nissinen A, Grobbee $D$, et al. Physical inactivity, depression, and risk of cardiovascular mortality. Med Sci Sports Exercise 2007;39(10):1693-1699.

15. Richardson C, Kriska A, Laniz P, Hayward R. Physical activity and mortality across cardiovascular disease risk groups. Med Sci Sports Exercise 2004;36(II):1923-1929.

16. Stewart K. Role of exercise training on cardiovascular disease in persons who have type 2 diabetes and hypertension. Cardiol Clin 2004;22:569-586.

17. Ortiz-Rodríguez SP, Torres-Mejía G, Mainero-Ratcheloux F, ÁngelesLlerenas A, López-Caudana AE, Lazcano-Ponce E, et al.Actividad física y riesgo de cáncer de mama en mujeres mexicanas. Salud Pub Mex 2008;50:126-135

18. Hernández B, De Haene J, Barquera S, Monterrubio E, Rivera J, Shamah $\mathrm{T}$, et al. Factores asociados con la actividad física en mujeres mexicanas en edad reproductiva. Rev Panam Salud Publica 2003; I4(4):235-245.

19. Wilson P, D'Agostino R, Levy D, Belanger A, Silbershatz H, Kannel W. Prediction of coronary heart disease using risk factor categories. Circulation 1998;97:1837-1847.

20. International Lipid Information Bureau (ILIB). Guías ILIB para el diagnóstico y manejo de las dislipidemias en Latinoamérica. Resumen Ejecutivo. Lipid Digest Latinoam 2002;8(I):2-8.

21. Salmerón-Castro J,Arillo-Santillán E, Campuzano-Rincón JC, LópezAntuñano $\mathrm{Fl}$, Lazcano-Ponce EC. Tabaquismo en profesionales de la salud del Instituto Mexicano del Seguro Social, Morelos. Salud Pub Mex 2002;44(Suppl I): 67-75.

22. López-Caudana AE,Téllez-Rojo Solís MM, Hernández-Ávila M, Clark P, Juárez-Márquez SA, Lazcano-Ponce EC, et al. Predictors of bone mineral 
density in female workers in Morelos State, Mexico. Arch Med Res 2005;35: $17 \mid-180$

23. Hernández-Avila M, Romieu I, Parra-Cabrera S, Hernández-Avila JE, Madrigal H,Willett W.Validity and reproducibility of a food frequency questionnaire to assess dietary intake of women living in Mexico City. Salud Pub Mex 1998;40:133-140.

24. Ainsworth B, Haskell W,Whitt M, Irwin M, Swartz A, Strath S, et al. Compendium of physical activities: an update of activity codes and MET intensities. Med Sci Sports Exerc 2000;32(Suppl 9):498-5I6.

25. World Health Organization. Physical status: the use and interpretation of anthropometry. Geneva:WHO, 1995.

26. Willet W, Stampfer M. Implications of total energy intake for epidemiologic analyses. En: Nutritional epidemiology. New York: Oxford University, I998; vol.20:273-30I.

27. Cañón L, Díaz N, Cruces E, Nieto T, Garrote T, Buitrago F. Capacidad predictiva, comparación y consecuencias clínicas de las tablas de Framingham-Wilson y REGICOR en personas atendidas en un centro de salud de Badajoz. Rev Esp Salud Pública 2007;81:353-364.

28. Mendivil C, Sierra I, Pérez C.Valoración del riesgo cardiovascular global y prevalencia de dislipidemias según los criterios del NCEP-ATPIII en una población adulta de Bogotá, Colombia. Clin Invest Arterioscl 2004;16(3):99-107.
29. Inelmen EM, Sergi G, Coin A, Miotto F, Peruzza S, Enzi G. Can obesity be a risk factor in elderly people? Obes Rev 2003;4:I47-I55.

30.Andresdottir M, Sigurdsson G, Sigvaldason H, Gudnason V. Fifteen percent of myocardial infarctions and coronary revascularizations explained by family history unrelated to conventional risk factors. Eur Heart J 2002;23:1655-1663.

31. Ciruzzi M, Schargrodsky H, Pramparo P, Rivas E, Rodríguez L, De la Noval R, et al. Attributable risks for acute myocardial infarction in four countries of Latin America. Medicina (B Aires) 2003;63:697-703.

32. Pi-Sunyer F.The relation of adipose tissue to cardiometabolic risk. Clin Cornerstone 2006;(Suppl 4): I4-23.

33. Acosta-Cázares B,Aranda-Álvarez JG, Reyes-Morales H. Patrones de actividad física de la mujer y del hombre. Rev Med Inst Mex Seguro Soc 2006;44(Suppl I):79-86.

34. The World Health Report 2002. Reducing risks, promoting healthy life. Geneva:World Health Organization 2002.

35. Ramírez-Vargas E,Arnaud-Viñas MR, Delisle H. Prevalence of metabolic syndrome and associated lifestyles in adults males from Oaxaca, Mexico. Salud Publica Mex 2007;49(2):95-102. 